\title{
Performance Evaluation of Adaptive Residual Interpolation, a Tool for Inter-layer Prediction in H.264/AVC Scalable Video Coding
}

\author{
Koen De Wolf, Davy De Schrijver, Jan De Cock, Wesley De Neve, \\ and Rik Van de Walle \\ Ghent University - IBBT \\ Department of Electronics and Information Systems - Multimedia Lab \\ Gaston Crommenlaan 8 bus 201, B-9050 Ledeberg-Ghent, Belgium \\ koen. dewolf@ugent. be
}

\begin{abstract}
Inter-layer prediction is the most important technique for improving coding performance in spatial enhancement layers in Scalable Video Coding (SVC). In this paper we discuss Adaptive Residual Interpolation (ARI), a new approach to inter-layer prediction of residual data. This prediction method yields a higher coding performance. We integrated the ARI tool in the Joint Scalable Video Model software. Special attention was paid to the CABAC context model initialization. Further, the use, complexity, and coding performance of this technology is discussed. Three filters were tested for the interpolation of lower-layer residuals: a bi-linear filter, the H.264/AVC 6-tap filter, and a median filter. Tests have shown that ARI prediction results in an average bit rate reduction of $0.40 \%$ for the tested configurations without a loss in visual quality. In a particular test case, a maximum bit rate reduction of $10.10 \%$ was observed for the same objective quality.
\end{abstract}

\section{Introduction}

Scalable Video Coding (SVC) adds an extra dimension to traditional video compression schemes: adaptivity. SVC bit streams can be adapted by reducing the spatial resolution and/or temporal resolution and/or the quality level of the decoded video. This enables multimedia content providers to cope with the heterogeneity of networks and multimedia devices. Driven by standardization efforts of the Joint Video Team (JVT) - formed by the Moving Picture Experts Group (MPEG) and the ITU-T Video Coding Experts Group (VCEG), SVC is a hot topic in the domain of video coding standardization.

In this paper, we discuss and evaluate the novel concept of Adaptive Residual Interpolation (ARI), which is a new approach in scalable video coding for interlayer prediction of residual data. The use of this tool results in bit rate savings without a loss in visual quality. A complexity and coding performance analysis is presented. Further, we elaborate on the signaling of this prediction method in the bit stream using Context-based Adaptive Binary Arithmetic Coding (CABAC). 
This paper is organized as follows. First, we introduce H.264/AVC Scalable Video Coding [1], focusing on the layered structure of this coding scheme and on the inter-layer residual prediction. The subsequent section describes the concept of ARI, its use, and its computational complexity. The conducted tests, the used interpolation filters, the initialization of the CABAC context model, and the coding performance are discussed in Sect. 4 Finally, this paper is concluded in Sect. 5 .

\section{H.264/AVC Scalable Video Coding}

In this paper, we focus on the Joint Scalable Video Model (JSVM) as proposed by the JVT. In that context, scalability is formally defined in 2] as a functionality for the removal of parts of the coded video bit stream while achieving a RateDistortion (R-D) performance at any supported spatial, temporal, or Signal-toNoise-Ratio (SNR) resolution that is comparable to the single-layer H.264/AVC coding scheme [3] at that particular resolution. In this requirements document, a coding efficiency penalty of $10 \%$ in bit rate for the same perceptual quality is set as an upper limit. Based on this definition, we can identify three main types of scalability: spatial, temporal, and SNR scalability. Spatial scalability means that it should be possible to decode the input video at lower spatial resolutions. Temporal scalability means that frames can be dropped in the bit stream. This implies that not all encoded frames will be decoded, resulting in a lower frame rate of the decoded video sequence. Finally, SNR scalability means that the bit stream can be truncated in order to reduce the bit rate. Undeniably, this will also result in a decrease of the visual quality. These types of scalability can be embedded in the bit stream individually or as combinations, based on the requirements of the target application.

SVC can be classified as a layered video specification based on the single-layer H.264/AVC specification. Enhancement Layers (ELs) are added which contain information pertaining to the embedded spatial and SNR enhancements. Similar single-layer prediction techniques as in H.264/AVC are applied, in particular intra and motion-compensated prediction. However, additional inter-layer prediction mechanisms have been added for the minimization of redundant information between the different layers.

\subsection{JSVM Structure}

As mentioned, the JSVM is a layered extension of the H.264/AVC video coding specification. The structure of a possible JSVM encoder is shown in Fig. 1] In this figure, the original input video sequence is downscaled in order to obtain the pictures for all different spatial layers (resulting in spatial scalability). On each spatial layer, a motion-compensated pyramidal decomposition is performed taking into account the characteristics of each layer. This temporal decomposition results in a motion vector field on the one hand and residual texture data on the other hand. This information is coded by using similar techniques as in H.264/AVC extended with progressive SNR refinement features. 


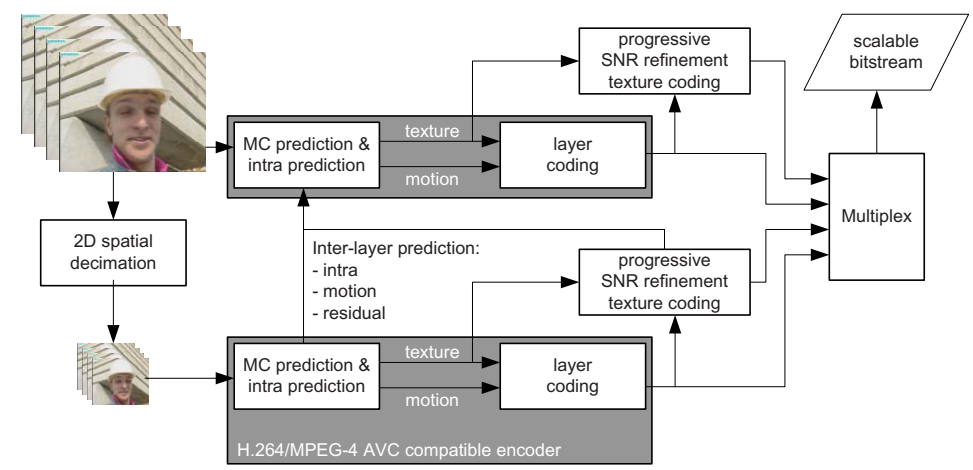

Fig. 1. SVC encoder structure supporting two spatial layers and progressive SNR refinement 4

A typical JSVM bit stream contains hierarchical layers. A JSVM-aware adaptation engine is capable of extracting only these layers that are necessary for decoding the video at a targeted (possibly reduced) spatial resolution, frame rate, and/or bit rate.

Several methods that allow the reuse of coded information among different spatial resolution layers are under investigation by the JVT. In particular, the layered structure of the JSVM allows the reuse of motion vectors, residual data, and intra texture information of lower spatial and SNR layers for the prediction of higher-layer pictures in order to reduce inter-layer redundancy. In the next section, we elaborate on these inter-layer prediction methods. Also, note that other extended spatial scalability modes are considered by the JVT, such as cropping and non-dyadic scaling. In this paper, we will focus on inter-layer prediction using residual pictures from lower layers.

\subsection{Inter-layer Residual Prediction}

In SVC, residual information of inter-picture coded macroblocks (MBs) from a lower resolution layer can be used for the prediction of the residual of the current layer. A flag, indicating whether inter-layer residual prediction is used, is transmitted for each MB of an enhancement layer. When residual prediction is applied, the lower resolution layer residuals of the co-located sub-MBs are blockwise upsampled using a bi-linear filter with constant border extension. Doing this, only the difference between the residual of the current layer obtained after motion compensation (MC) and the upsampled residual of a lower resolution layer is coded. In Fig. 2, the picture reconstruction scheme using inter-layer residual prediction of the decoder is shown.

At the encoder side, the decoded and upsampled lower layer residuals are subtracted from the original image in the current layer preceding the Motion Estimation (ME) stage. This is illustrated in Fig. 3. By applying residual prediction in advance of $\mathrm{ME}$, we are sure to obtain the most efficient motion vectors 


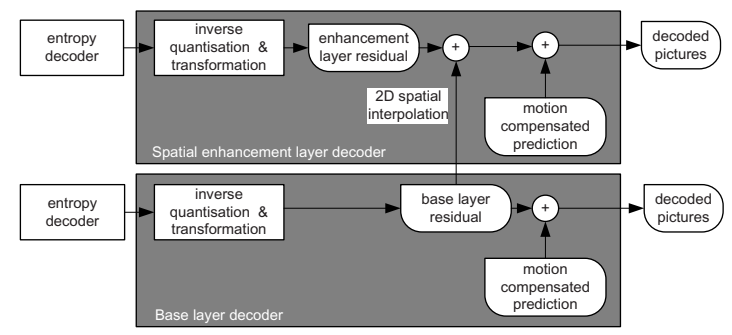

Fig. 2. Structure of the decoder using residual prediction

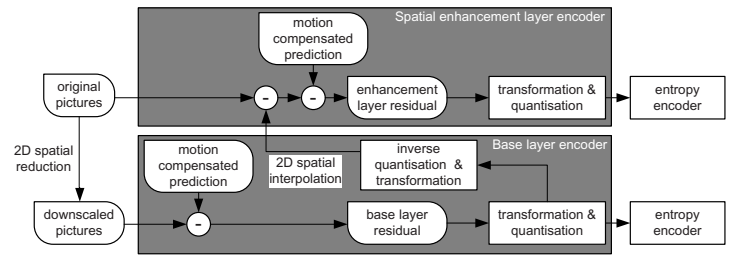

Fig. 3. Prediction of enhancement layer residual at the encoder

resulting in lower magnitudes in the enhancement layer residual which will result in a higher compression efficiency.

Inter-layer residual prediction has shown to be useful when the motion vectors of a block are aligned with the motion vectors of the corresponding block in a lower spatial resolution layer. This can be explained by the likeliness that after motion compensation, the residuals for those blocks will show a high resemblance.

\section{Adaptive Residual Interpolation}

\subsection{Definition}

ARI can be seen as an extension to the inter-layer residual prediction technique that is incorporated in the JSVM, (as explained in Sect. 2.2). In 5], we have presented ARI as a prediction method applied in the context of SVC which evaluates multiple interpolation filters used for the upsampling of lower layer residuals. This evaluation is performed on a MB basis in an R-D optimized sense. The latter means that a cost function is used to determine which filter should be used.

In [6], we have shown that the use of multiple pre-defined interpolation filters for the upsampling of base layer residuals, results in a better prediction of the enhancement layer residuals. As a result, the magnitudes of these remaining residuals are lower. The applied filter was chosen on a picture basis. Moreover, lower bit rates and Peak Signal-to-Noise Ratio (PSNR) gains can be achieved when the applied residual interpolation filter is changed on a MB basis, hereby 
selecting the best filter out of multiple pre-defined interpolation filters for that particular MB [5].

\subsection{Computational Complexity}

Decoder Complexity. Adding ARI as an extra prediction mode results in a limited change of complexity at the decoder side. When residual prediction is used for a particular MB, its corresponding base layer residual must be upsampled anyhow. So, the minor complexity introduced by ARI originates from the complexity of the filters being used. In particular, the computational complexity is reduced when the applied filters are less complex than the bi-linear filter (which is defined in the JSVM specification) or that the complexity is increased when using more complex interpolation filters.

Encoder Complexity. The complexity is significantly increased when all modes for the coding of a enhancement layer MB are evaluated at the encoder side in order to obtain an R-D optimized coding decision. This is caused by the fact that, when no fast mode-decision algorithm is used, a distinct ME needs to be performed for each interpolation filter and this for all possible coding modes. In general, implementations of contemporary video coding specifications will use fast mode-decision and fast ME algorithms to cope with the high computational complexity. These algorithms can be modified in order to support ARI. For example, by relying on hierarchical search algorithms and previously made coding decisions. For off-line scenarios, this extra complexity shouldn't be a burden.

\section{Tests and Results}

\subsection{Test Setup}

For the performance evaluation of ARI in terms of coding efficiency, we integrated the ARI tool in the JSVM software. We have implemented two additional filters next to the bi-linear filter which is already incorporated in the current JSVM specification (version 5). We have chosen the 6-tap filter already specified in both H.264/AVC and the JSVM (used there for sub-pixel ME) in combination with a median filter. A description of these filters is given in Sect.4.2. Note that the interpolation is done on $4 \times 4$-blocks with constant border extension as specified in the JSVM.

In order to limit the encoder complexity, we limited the number of filters that can be chosen for residual interpolation, to two. In this test, we used two filter combinations: bi-linear \& H.264/AVC 6-tap filter (combination A) and H.264/AVC 6-tap \& median filter (combination B). Five CIF-resolution test sequences of 300 pictures at $30 \mathrm{~Hz}$ with different motion characteristics were used: Crew, Foreman, Mobile \& Calender, Mother \& Daughter, and Stefan. A downsampled version was generated using the 11-tap FIR JSVM downsampling filter. This results in a base layer at QCIF resolution and a CIF spatial enhancement layer. We used 4 fixed Quantization Parameter (QPs) for the Base Layer 
$\left(Q P_{B L}\right)$ and the Enhancement Layer $\left(Q P_{E L}\right): 12,18,24,30$. This leads to 16 tested QP-combinations. These QP-combinations are chosen to cover a broad range of possible applications. ARI is used for the prediction of $\mathrm{P}$-pictures only. The achievable coding gains when ARI is used for B-pictures, are minimal. This is due to the fact that for most MBs in B-pictures no additional residual is coded. As such, enabling ARI in B-pictures will result in the coding of an extra flag without enhancing the coded residual.

\subsection{Definition and Complexity of the Tested Interpolation Filters}

AVC 6-tap Filter. For the derivation of pixel values at half-sample positions, we refer to the H.264/AVC specification and an overview paper by Wiegand et al. 7]. An in-depth complexity analysis with respect to this filter is performed in 8 .

Median Filter. In Fig. 4, the positions labeled with upper-case letters represent the signal at full-sample locations inside a two-dimensional block of residual samples. The signals at half-sample locations (labeled with lower-case letters) are derived as follows.

1. The value of each sample labeled with double lower-case letters is determined by the median of the neighboring full-sample values; e.g., cc $=\operatorname{Median}(\mathrm{D}$, E, H, I);

2. The value of each sample labeled with a single lower-case letter is determined by the median of the two neighboring full-sample values and the two neighboring half-sample values as derived in Step 1; e.g., a = Median(D, E, aa, cc).

For samples at the border of a $4 \times 4$ block, the complexity of the sorting algorithm can be reduced when constant border extension is used for the construction of the samples outside the $4 \times 4$ block. In particular, in Fig. 4 , the value for $x$ is derived as

$$
\operatorname{Med}\left(\frac{(A+B)}{2}, A, B, a a\right) \text {. }
$$

This can be simplified as follows:

Let $A<B$,

$$
x= \begin{cases}\frac{3 A+B}{4} & \text { if } a a<A, \\ \frac{A+3 B}{4} & \text { if } B<a a, \\ \frac{2 a a+A+B}{4} & \text { if } a a \in[A . . B] .\end{cases}
$$

\subsection{ARI Signaling}

For each MB, the choice whether residual prediction is being used, has to be signaled. For ARI, an additional syntax element denoting which interpolation 


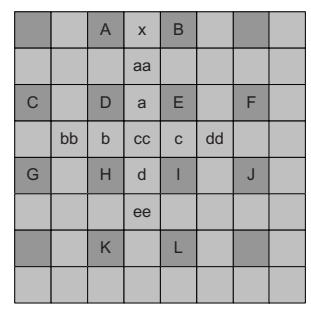

Fig. 4. Full-sample positions (dark-shaded blocks with upper-case letters) and halfsample positions (light-shaded blocks with lower-case letters)

filter is being applied, needs to be coded for each MB. In our test, we limited the number of filters to two. As such, this syntax element can take 0 or 1 as value. This syntax element (symbol) is coded using a CABAC entropy coder. Within this context-based coder, a model probability distribution is assigned to the symbols that need to be coded. Furthermore, this coding engine adaptively changes the state of the context models (i.e., probability model distribution) based on symbols already coded.

Such a context state of model $\gamma$ is determined by two parameters: the probability of the Least Probable Symbol (LPS) $\sigma_{\gamma}$ and the Most Probable Symbol (MPS) $\varpi_{\gamma}$. For the ARI context, MPS denotes which of both filters is most probably being used. The LPS probability indicates the probability of the other filter being used for the current context state.

The initial state for the context is determined by two parameters $\left(\mu_{\gamma}, \nu_{\gamma}\right)$. These parameters are used to derive $\varpi_{\gamma}$ and $\sigma_{\gamma}$, hereby taking into account the $\mathrm{QP}$ of the current slice For a detailed explanation on context state transition and context initialization, we refer to [9].

For this ARI context, $\mu_{\gamma}$ and $\nu_{\gamma}$ were deduced using linear regression on the results of a training set (115 tested configurations) that have been used to fit the different initial probability states for both filter combinations (i.e., combinations A and B). In Fig. 5, a QP-based probability of median filter usage for filter combination B (i.e., H.264/AVC 6-tap \& median filter) is given. This probability is plotted against the QP of the enhancement layer. In this graph, we make a distinction between the different QPs of the base layer. Linear regression lines are drawn for all tested configurations with the same $Q P_{B L}$. A linear regression line that takes all tested configurations into account is also drawn. It is clear from the graph that the $\mathrm{QP}$ of the base layer has an impact on the behavior of this regression line and thus on the initial probability $\sigma$.

Context model initialization in CABAC was designed for the single-layer H.264/AVC coding specification. As such, a model can not be initialized by using the QP of the base layer. Therefore the context model initialization for inter-layer prediction tools in the JSVM is sub-optimal. This shortcoming can 


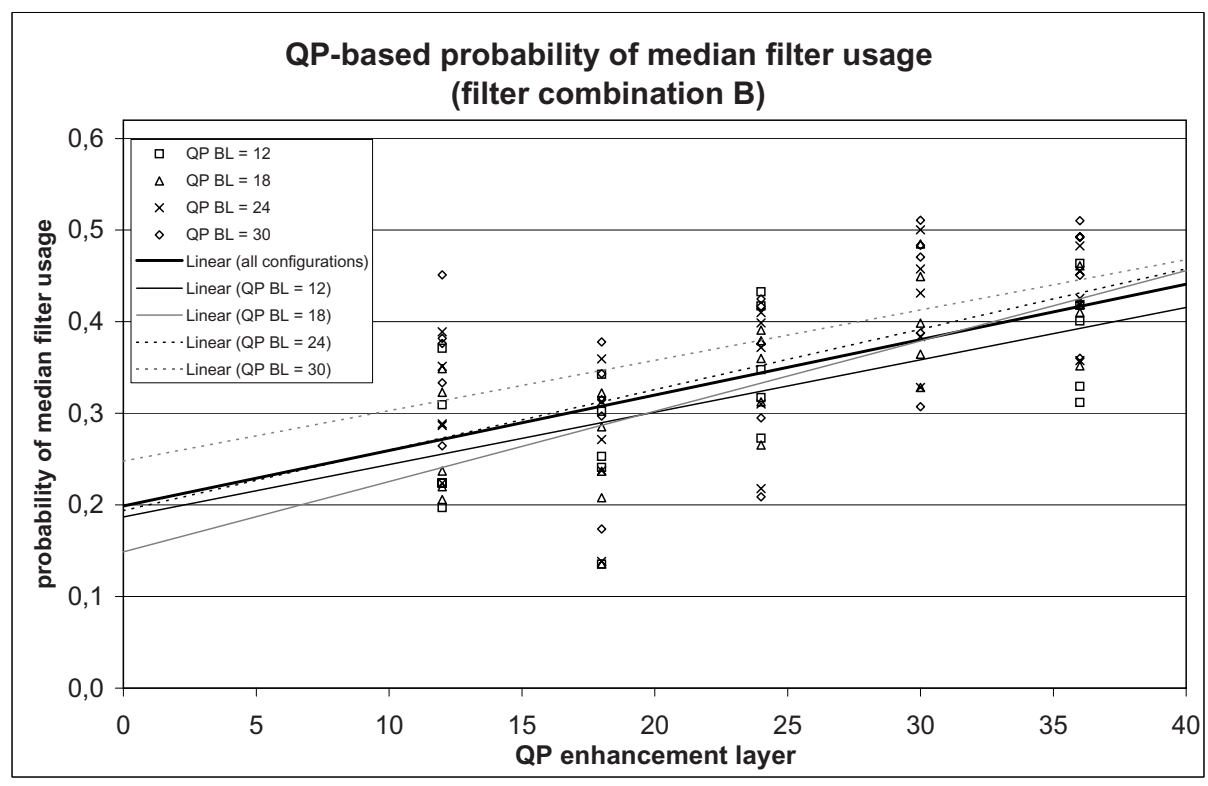

Fig. 5. QP-based probability of median filter usage for filter combination B

somehow be circumvented by using cabac_init_idd 1 . Doing so, three different initialization parameter pairs $\left(\mu_{\gamma}, \nu_{\gamma}\right)$ can be defined per context model. The values of these pairs can be chosen, taking into account the base layer QP.

\subsection{Results}

The coding performance of the presented inter-layer residual prediction tool is evaluated using Bjøntegaard Delta Bit Rate (BD-BR) and Bjøntegaard Delta PSNR (BD-PSNR) [10, which are respectively the average bit rate difference and the PSNR difference between the original residual prediction algorithm (explained in Sect. 2.2) and the proposed ARI algorithm. BD-BR and BD-PSNR are derived from simulation results for each fixed $Q P_{B L}$ in combination with varying $Q P_{E L}=28,32,36,40$.

From Table 1] we observe that the BD-PSNR of the tested configurations with ARI enabled, shows a slight gain (less than $0.1 \mathrm{~dB}$ ). From Table 2, we see that the highest bit rate reductions are achieved for low $Q P_{B L}$ when filter combination $\mathrm{B}$ is used. This can be explained by the fact that when $Q P_{\mathrm{BL}}$ is low, more information will be present in the coded residual, which can be used for inter-layer residual prediction on the one hand and the median filter preserves

\footnotetext{
$\overline{1}$ cabac_init_idc specifies the index for determining the initialization table used in the initialization process for context variables. The value of cabac_init_idc shall be in the range of 0 to 2 , inclusive. [3]
} 
Table 1. ARI performance: BD-PSNR (dB) for filter combinations $A$ and $B$

\begin{tabular}{c||cc|cc|cc|cc|cc}
$Q P_{B L}$ & Crew & Foreman & \multicolumn{2}{|c|}{$\begin{array}{l}\text { Mobile \& } \\
\text { Calender }\end{array}$} & \multicolumn{3}{|c|}{$\begin{array}{l}\text { Mother } \\
\text { Daughter }\end{array}$} & Stefan \\
\hline & $\mathrm{A}$ & $\mathrm{B}$ & $\mathrm{A}$ & $\mathrm{B}$ & $\mathrm{A}$ & $\mathrm{B}$ & $\mathrm{A}$ & $\mathrm{B}$ & $\mathrm{A}$ & $\mathrm{B}$ \\
\hline 12 & 0.05 & 0.07 & 0.02 & 0.03 & 0.02 & 0.03 & 0.06 & 0.11 & 0.03 & 0.04 \\
18 & 0.03 & 0.04 & 0.02 & 0.02 & 0.02 & 0.02 & 0.04 & 0.05 & 0.03 & 0.02 \\
24 & -0.02 & 0.03 & 0.02 & 0.01 & 0.03 & 0.02 & 0.02 & 0.02 & 0.03 & 0.02 \\
30 & 0.01 & 0.01 & 0.01 & 0.01 & 0.02 & 0.02 & 0.02 & 0.02 & 0.01 & 0.00 \\
\hline
\end{tabular}

Table 2. ARI performance: BD-BR (\%) for filter combinations $A$ and $B$

\begin{tabular}{|c|c|c|c|c|c|c|c|c|c|c|}
\hline \multirow[t]{2}{*}{$Q P_{B L}$} & \multicolumn{2}{|c|}{ Crew } & \multicolumn{2}{|c|}{ Foreman } & \multicolumn{2}{|c|}{$\begin{array}{l}\text { Mobile \& } \\
\text { Calender }\end{array}$} & \multicolumn{2}{|c|}{$\begin{array}{l}\text { Mother \& } \\
\text { Daughter }\end{array}$} & \multicolumn{2}{|c|}{ Stefan } \\
\hline & & & A & B & $\mathrm{A}$ & B & $\mathrm{A}$ & B & $\mathrm{A}$ & B \\
\hline 12 & 00 & 1.15 & -0.35 & -0.55 & -0.25 & -0.28 & -1.29 & -2.01 & -0.3 & 0.30 \\
\hline 18 & -0.49 & -0.57 & -0.32 & -0.32 & -0.20 & -0.15 & -0.76 & -0.92 & -0.32 & -0.23 \\
\hline 24 & -0.28 & -0.37 & -0.22 & -0.20 & -0.23 & -0.16 & -0.38 & -0.41 & -0.29 & -0.22 \\
\hline 30 & -0.08 & -0.08 & -0.16 & -0.08 & -0.16 & -0.15 & -0.38 & -0.35 & -0.08 & -0.04 \\
\hline
\end{tabular}

borders in the residuals, whereas the bi-linear and H.264/AVC 6-tap filters rather smoothen these borders on the other hand.

Due to space limitations, we are unable to publish all results in detail. However, we have observed a clear relation between bit rate reduction and the values of $Q P_{\mathrm{BL}}$ and $Q P_{\mathrm{EL}}$. When the $\mathrm{BL}$ is less quantized than the EL, the bit rate reduction increases when the difference between $Q P_{\mathrm{BL}}$ and $Q P_{\mathrm{EL}}$ increases.

The motion characteristics of the coded video sequences also have an important impact on the bit rate reduction. ARI proves to be very useful for lowmotion sequences, such as the Mother \& Daughter sequence. For the coding of this sequence, a maximum bit rate reduction of $10.10 \%$ is achieved when filter combination B is used with $Q P_{\mathrm{BL}}=24$ and $Q P_{\mathrm{EL}}=36$ (not shown in this paper). However, complex-motion sequences, such as Mobile\& Calender, still benefit from ARI.

The average bit rate reduction for the tested configurations is $0.4 \%$ with an average PSNR increase of $0.03 \mathrm{~dB}$.

\section{Conclusion and Future Work}

In this paper, we have presented ARI as a tool for inter-layer residual prediction in SVC. The use, computational complexity, CABAC context initialization, and 
coding performance of the tested filters were discussed. This tool proves to be especially useful for coding low-motion sequences when the combination of the 6-tap filter and the median filter is used for the interpolation of lower-layer residuals. Moreover, complex-motion sequences also benefit from ARI.

We have observed that impact of the newly introduced syntax element is not negligible. In future work, we will investigate a combined signaling of this syntax element in the slice header and MB header in order to further improve the coding performance of this inter-layer prediction tool.

\section{Acknowledgements}

The research activities that have been described in this paper were funded by Ghent University, the Interdisciplinary Institute for Broadband Technology (IBBT), the Institute for the Promotion of Innovation by Science and Technology in Flanders (IWT), the fund for Scientific Research-Flanders (FWO-Flanders), the Belgian Federal Science Policy Office (BFSPO), and the European Union.

\section{References}

1. Reichel, J., Schwarz, H., Wien, M.: Joint scalable video model JSVM 5, doc. JVTR202 (2006)

2. ISO/IEC, JTC1/SC29/WG11: Applications and requirements for scalable video coding. ISO/IEC JTC1/SC29/WG11 N6880 (2005)

3. ITU-T, ISO/IEC JTC 1: Advanced video coding for generic audiovisual services, ITU-T Rec. H.264 and ISO/IEC 14496-10 AVC (2003)

4. Schwarz, H., Marpe, D., Wiegand, T.: Basics concepts for supporting spatial and SNR scalability in the scalable H.264/MPEG4-AVC extension. In: Proceedings of IEEE IWSSIP (2005)

5. De Wolf, K., De Schrijver, D., De Neve, W., Van de Walle, R.: Adaptive Residual Interpolation: a tool for efficient spatial scalability in digital video coding. In: Proceedings of International Conference on Image Processing, Computer Vision \& Pattern Recognition (IPCV'06). vol. 1, pp. 131-137, Las Vegas (2006)

6. De Wolf, K., De Sutter, R., De Neve, W., Van de Walle, R.: Comparison of prediction schemes with motion information reuse for low complexity spatial scalability. In: Proceedings of SPIE/Visual Communications and Image Processing. vol. 5960, pp. 1911-1920, Beijing (2005)

7. Wiegand, T., Sullivan, G., Bjøntegaard, G., Luthra, A.: Overview of the H.264/AVC video coding standard. IEEE Trans. CS Video Technology 13, 560 $576(2003)$

8. Horowitz, M., Joch, A., Kossentini, F., Hallapuro, A.: H.264/AVC baseline profile decoder complexity analysis. IEEE Trans. CS Video Technology 13, 704-716 (2003)

9. Marpe, D., Wiegand, T., Schwarz, H.: Context-based adaptive binary arithmetic coding in the H.264/AVC video compression standard. IEEE Trans. CS Video Technology 13, 620-636 (2003)

10. Bjøntegaard, G.: Calculation of average PSNR difference between RD-curves, VCEG-M33 (2001) 